\title{
Steam Generator Level Control with an Observer-Based Algebraic Approach
}

\author{
Günyaz Ablay \\ Electrical \& Electronics Engineering, Abdullah Gül University, Kayseri, Turkey \\ gunyaz.ablay@agu.edu.tr
}

\begin{abstract}
Level control systems are used to maintain the steam generator water level within the prescribed narrow limits and to provide constant supply of steam during power demand variations. The current level control systems are often found to be unsatisfactory during startup and low power operations. A robust nonlinear estimator-based optimal algebraic control is developed for level control systems to solve the water level tracking problem during power demand variations. It is shown that the proposed control provides an optimal and robust water level tracking with a single automatic controller over the complete range of power operation in the presence of plant uncertainties and noisy measurements.
\end{abstract}

\section{Introduction}

Steam generator level control systems are used extensively throughout the nuclear power plants and process industry to control boiling water level and supply high quality steam. Variations of the water level in the steam generators can cause certain problems including flooding of steam purification equipment and damaging turbine blades in case of too high water level, and reduction in efficiency of the recirculation function if the water level is too low. It is necessary to keep water level in the range of prescribed narrow limits for providing a smooth and uninterrupted plant operation in the face of varying power demand.

Steam generators are highly complex, nonlinear, and timevarying systems because of fluctuations in plant parameters with the varying operating conditions. Several studies have shown that the steam generator level control system is a major contributor to plant unavailability, about $25 \%$, mainly due to the poor control of the steam generator water level [1], [2]. For this reason, the steam generator level control designs spread over the last thirty years in the literature. An adaptive control scheme was given in [1], PID type controllers incorporated with feedforward controllers were reported in [3], [4], [5], the $\mathrm{H}_{\infty}$ controller was designed in [6], a gain scheduled $\mathrm{L}_{2}$ controller was designed in [7], the model predictive controller were applied to steam generator in [8], the output feedback dissipation method was designed in [9], the linear quadratic regulator method was applied in [10], an adaptive observer based method was reported in [11], fuzzy and neurofuzzy controllers were given in [12], a data driven PI controller was reported in [13], an adaptive backstepping-based controller was designed in [14], and the sliding mode control was designed in [15], [16]. Most of these methods have considered either local operating conditions (specifically for low power mode) or piecewise linear regions to provide a level control mechanism. Theoretically, the above studies have achieved good results with some degree. From a practical point of view, the open problems include: (i) a single automatic control over the complete range of power operation, (ii) reduction in control equipment for lowering price but increasing reliability, and (iii) reduction in control algorithms and information load in the whole level control system.

The objective of this work is to develop a robust, efficient and simple robust estimator based optimal algebraic control strategy for the steam generator level system from start-up to full load conditions. The main advantages of this approach are optimal performance, low sensitivity to disturbances, easily implementable in practice, and utilization of the good features of the modern and classical control.

The organization of the research is as follows: Section 2 provides a background on steam generator level control systems. The approach to level control system design is given in Section 3. Section 4 provides the numerical results, and the conclusion can be drawn from this work is given in Section 5 .

\section{Steam Generators and Problem Formulation}

The steam generator is a heat exchanger which is used to transfer heat generated by the reactor to steam energy and one of the major components of the many nuclear power plants, e.g. pressurized water reactors (PWR). The power demand from the grid or users causes variations in the water level of the steam generator. The water level must be maintained within its specified lower and upper limits to avoid serious consequences including unintended plant shutdowns and system damage. Difficulties in keeping water level constant in steam generators during power demand variations arise from the nonlinear plant characteristics, time-varying parameters, constraints on the available control action, limits on the water level, and noisy level and flow measurements.

\subsection{Mathematical Model of the Steam Generators}

For control purposes and simulations, the following linear, time-varying parameter model has been widely used in describing the steam generator dynamics accurately [1]:

$$
\begin{aligned}
y(s)=\frac{K_{1}}{s}(u-q) & -\frac{K_{2}}{1+\tau_{2} s}\left(u-K_{4} q\right) \\
& +\frac{K_{3} s}{\tau_{1}^{-2}+4 \pi^{2} T^{-2}+2 \tau_{1}^{-1} s+s^{2}} u
\end{aligned}
$$

In (1), $u$ is the feedwater flow rate, $q$ is the steam flow rate, and $y$ is the water level (narrow range level). The term $K_{1} / s$ represents the mass capacity effect of the steam generator, which integrates the steam-feedwater flow difference to calculate the change in water level. The second term $K_{2} /\left(1+\tau_{2} s\right)$ represents the thermal negative effect caused by 
the swell-shrink phenomenon, which appears initially in the case of feedwater or steam flow rate changes. The last term is the mechanical oscillation effect caused by the inflowing feedwater to the steam generator. The power-dependent parameters $\tau_{1}(p), \tau_{2}(p)$ are the damping time constants, $T(p)$ is the mechanical oscillation period, and $K_{1}, K_{2}(p), K_{4}(p)$ and $K_{3}(p)$ are the magnitudes of the mass capacity effect, swellshrink phenomenon, and mechanical oscillation effect, respectively. Here, $p$ (in \% rated power) denotes the operating power which determines the values of all the parameters. The model parameters identified from experimental data in [1] are given in Table 1.

Table 1. Steam generator model parameters regarding to operating power [1].

\begin{tabular}{|c|c|c|c|c|c|c|c|c|}
\hline Power & \multicolumn{7}{|c|}{ Experimental Parameters } \\
\cline { 2 - 9 } $\begin{array}{c}\text { Level } \\
(\boldsymbol{p} \%)\end{array}$ & $\begin{array}{c}\boldsymbol{K}_{\mathbf{1}} \\
(\mathrm{mm} / \mathrm{kg})\end{array}$ & $\begin{array}{c}\boldsymbol{K}_{\mathbf{2}} \\
(\mathrm{mm}-\mathrm{s} / \mathrm{kg})\end{array}$ & $\begin{array}{c}\boldsymbol{K}_{\mathbf{3}} \\
(\mathrm{mm} / \mathrm{kg})\end{array}$ & $\boldsymbol{K}_{\mathbf{4}}$ & $\begin{array}{c}\boldsymbol{T} \\
(\mathrm{s})\end{array}$ & $\begin{array}{c}\boldsymbol{\tau}_{\mathbf{1}} \\
(\mathrm{s})\end{array}$ & $\begin{array}{c}\boldsymbol{\tau}_{\mathbf{2}} \\
(\mathrm{s})\end{array}$ & $\begin{array}{c}\boldsymbol{q} \\
(\mathrm{kg} / \mathrm{s})\end{array}$ \\
\hline $5 \%$ & 0.058 & 9.63 & 0.181 & 1.60 & 119.6 & 41.9 & 48.4 & 57.4 \\
\hline $15 \%$ & 0.058 & 4.46 & 0.226 & 1.90 & 60.5 & 26.3 & 21.5 & 180.8 \\
\hline $30 \%$ & 0.058 & 1.83 & 0.310 & 2.25 & 17.7 & 43.4 & 4.5 & 381.8 \\
\hline $50 \%$ & 0.058 & 1.05 & 0.215 & 2.80 & 14.2 & 34.8 & 3.6 & 660.0 \\
\hline $100 \%$ & 0.058 & 0.47 & 0.105 & 4.82 & 11.7 & 28.6 & 3.4 & 1434.7 \\
\hline
\end{tabular}

The water level is the balance between feedwater flow and steam flow under steady-state conditions. For this reason, one of the most commonly used reduced-order model is the mass capacity effect [17]

$$
y_{0}(s)=\frac{K_{1}}{s}(u-q)
$$

The swell-shrink phenomenon which appears in the case of feedwater or steam flow rate changes has strong effects under transient conditions. On the other hand, the effect of the mechanical oscillations is much smaller compared with the mass capacity effect and swell-shrink phenomenon as showed in [19]. Therefore, the steam generator model (1) can also be represented as a second order system by ignoring the effects of the mechanical oscillations. That is, in the state-space, the steam generator can be modeled by

$$
\left\{\begin{array}{l}
\dot{x}_{1}=K_{1}(u-q) \\
\dot{x}_{2}=-\frac{1}{\tau_{2}(p)} x_{2}-\frac{K_{2}(p)}{\tau_{2}(p)}\left(u-K_{4}(p) q\right) \\
y=x_{1}+x_{2}
\end{array}\right.
$$

where $x=\left[x_{1}, x_{2}\right]^{T}$ is the state vector, $u$ is the control input (feedwater flow), and $y$ is the system output (water level). The state vector $x_{1}$ (in $\mathrm{mm}$ ) represents the contribution to the water level due to total mass of water in steam generator, and $x_{2}$ (in $\mathrm{mm})$ represents the reverse dynamic effect related to swellshrink phenomenon (i.e. level increase (swell) instead of decrease following steam flow increase due to expansion of bubbles in the two-phase fluid, and vice-versa).

\section{Control System Design}

A control system must provide stability, robustness and good performance for steam generator level system in the presence of swell-shrink effect, mechanical oscillations and steam flow fluctuations. While it is difficult to obtain optimal performances under these conditions, a simultaneous algebraic approach which handles the denominator and numerator of the transfer function of a system independently can be used to design the characteristic polynomial of the closed loop system, which specifies stability, response and robustness requirements. The strength of the algebraic approach is that a simple, optimal and robust controller can be designed for any plant under practical limitations.

\subsection{Design of an Optimal Algebraic Control System}

The optimal algebraic approach combines the good features of the modern and classical control. An optimal controller is selected to meet stability and performance requirements. A block diagram for the optimal algebraic controller based system is shown in Fig. 1. The plant is described through the transfer function with numerator $N(s)$ and denominator $D(s)$. The algebraic controller is specified with the controller denominator $A(s)$, feedback numerator $B(s)$ and reference numerator $F(s)$.

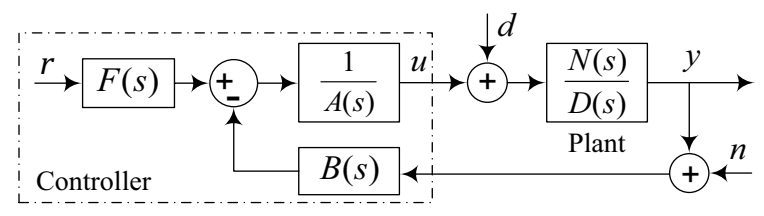

Fig. 1. Block diagram of the optimal algebraic control system.

The output of the closed-loop system can be expressed using Fig. 3 by

$$
y=\frac{N(s) F(s)}{P(s)} r+\frac{A(s) N(s)}{P(s)} d-\frac{N(s) B(s)}{P(s)} n
$$

where $y$ is the system output, $r$ is the reference signal, $d$ is the disturbance and $n$ is the measurement noise. Consider the transfer function of a linear time-invariant plant

$$
\frac{N(s)}{D(s)}=\frac{c_{z} s^{z}+\cdots+c_{1} s+c_{0}}{b_{m} s^{m}+\cdots+b_{1} s+b_{0}}
$$

where $z \leq m$. Considering Fig. 1, the objective is to develop a control scheme which forces the system output $y$ to follow a reference signal $r$ with desired performance specifications. The controller polynomials $A(s), B(s)$, and $F(s)$ can be given by

$$
\begin{aligned}
A(s) & =\sum_{i=0}^{p} l_{i} s^{i} \\
B(s) & =\sum_{i=0}^{q} k_{i} s^{i} \\
F(s) & =\left.(P(s) / N(s))\right|_{s=0}
\end{aligned}
$$

where $p$ and $q$ are the degrees of the controller denominator $\operatorname{deg}\{A(s)\}$ and feedback numerator $\operatorname{deg}\{B(s)\}$, respectively. If plant contains disturbance or noise, the controller polynomials are selected such that the effects of disturbance and noise are minimized, e.g. $\operatorname{deg}\{A(s)\}=\operatorname{deg}\{B(s)\}=m$. If the reference numerator $F(s)$ is selected as given in (13), the steady-state error of the controlled system becomes zero, and the closed loop transfer function turns out to be a type-1 system (no overshoot in 
the system response) so that a good closed-loop time response can be acquired. The denominator $P(s)$ is the characteristic polynomial given by

$$
\begin{aligned}
P(s) & =A(s) D(s)+B(s) N(s) \\
& =a_{n} s^{n}+\ldots+a_{1} s+a_{0}=\sum_{i=0}^{n} a_{i} s^{i}
\end{aligned}
$$

The main aim in the algebraic controller is to determine the coefficients of the characteristic polynomial $a_{i}$ to satisfy stability, response and robustness requirements of the plant. The performance specifications are given with stability index $\gamma_{i}$, equivalent time constant $\tau$, and stability limit $\gamma_{i}^{*}$. The relations between these parameters and the coefficients of the characteristic polynomial are as follows:

$$
\begin{aligned}
& \tau=a_{1} / a_{0} \\
& \gamma_{i}=a_{i}^{2} /\left(a_{i+1} a_{i-1}\right), \quad i=1,2, \ldots, n-1, \quad \gamma_{0}=\gamma_{n}=\infty \\
& \gamma_{i}^{*}=1 / \gamma_{i-1}+1 / \gamma_{i+1}
\end{aligned}
$$

One significant feature of the algebraic controller is that the stability index (or coefficient ratio) $\gamma_{i}$ can be assigned optimally (or arbitrarily) to solve the design problem. An optimal standard form for the stability index is given by [21]

$$
\gamma_{1}=2.5, \gamma_{i}=2, \quad i=2, \ldots, n-1
$$

From the relationship given in (10) and the optimal standard form of the stability index (11), the coefficients of (9) can easily be calculated as follows

$$
\begin{aligned}
& a_{1}=a_{0} \tau \\
& a_{i}=\frac{a_{0} \tau^{i}}{\gamma_{i-1} \gamma_{i-2}^{2} \gamma_{i-3}^{3} \cdots \gamma_{2}^{i-2} \gamma_{1}^{i-1}}, \text { for } i=2, \cdots, n
\end{aligned}
$$

The standard form (11) is recommended in order to get optimal stability and response performances. The equivalent time constant $\tau$ is selected to satisfy the settling time $t_{s}$ requirement. The $\tau$ characterizes the speed of a closed-loop system with denominator $P(s)$, and if the standard form of the stability index is used, the shortest settling time is obtained, i.e.

$$
t_{s}=2.5 \tau \sim 3 \tau
$$

On the other hand, the equivalent time constant $\tau$ can always be adjusted through adjusting the stability index (11) to satisfy the design requirements depending on the plant characteristics. The stability of the closed loop system is determined from RouthHurwitz criterion for low-order characteristic polynomials, and for higher order systems, the stability criterion is given by [23]

$$
\gamma_{i}>1.12 \gamma_{i}^{*}, \quad i=2,3, \ldots, n-2
$$

and the sufficient condition for instability is given by

$$
\gamma_{i} \gamma_{i+1} \leq 1 \text {, for some } i=1,2, \ldots, n-2
$$

In order to guarantee the stability of the characteristic polynomial based on stability and robustness conditions, all $\gamma_{i}$ 's are usually chosen within interval $\gamma_{i} \in[1.5,4]$.

\subsection{A Practical Controller for the Level Control System}

A simple algebraic controller can be designed by using the mass capacity effect model since the water level is the balance between feedwater flow and steam flow under steady-state conditions. The other parts of the steam generator model, swell-shrink phenomenon and mechanical oscillation effect, do have negligible effects on the system response under steadystate and high power conditions. Thus, by using the model (2), the numerator and denominator of the steam generator can simply be written as

$$
\begin{aligned}
& N(s)=K_{1} \\
& D(s)=s
\end{aligned}
$$

where $K_{1}=0.058$ (see Table 1). Following the control design approach given in Section 3.1, the polynomials of the optimal algebraic controller are designed as follows:

$$
\begin{aligned}
& A(s)=l_{1} s \\
& B(s)=k_{1} s+k_{0} \\
& F(s)=k_{0}
\end{aligned}
$$

The main aim in such selections is to eliminate the effects of the disturbance, i.e. steam flow rate. From (16) and (17), the characteristic polynomial of the closed-loop system in the form of (9) can be obtained as

$$
P(s)=l_{1} s^{2}+k_{1} K_{1} s+k_{0} K_{1}
$$

To determine the controller parameters, $l_{1}, k_{1}$ and $k_{0}$, the standard design specifications will be used, i.e. $\gamma_{1}=2.5$. The desired setting time for a steam generator is about $t_{s}=300$ seconds, which leads to a time constant of $\tau=100$ seconds by using the relation (13). Then the control parameters can easily be found as $k_{0}=1, k_{1}=100$ and $l_{1}=232$.

The stability of the steam generator plant under optimal algebraic controller and reduced-order model (2) can be evaluated as follows. If we consider state-space representation of plant (1) and control (17) with state variables $x=$ $\left[x_{1}, x_{2}, x_{3}, x_{4}\right]^{T}$ where $x_{1}=$ mass capacity effect, $x_{2}=$ swellshrink effect, $x_{3}=$ mechanical oscillation effect and $x_{4}=$ timederivative of $x_{3}$, the augmented closed-loop system under the optimal algebraic controller can be written as

$$
\dot{x}=(A-B K) x
$$

where

$x=\left[\begin{array}{l}x_{1} \\ x_{2} \\ x_{3} \\ x_{4} \\ \sigma\end{array}\right], \quad A=\left[\begin{array}{ccccc}0 & 0 & 0 & 0 & 0 \\ 0 & -\tau_{2}^{-1} & 0 & 0 & 0 \\ 0 & 0 & -2 \tau_{1}^{-1} & 1 & 0 \\ 0 & 0 & -\tau_{1}^{-2}-4 \pi^{2} T^{-2} & 0 & 0 \\ 1 & 0 & 0 & 0 & 0\end{array}\right], B=\left[\begin{array}{c}K_{1} \\ -K_{2} \tau_{2}^{-1} \\ K_{3} \\ 0 \\ 0\end{array}\right], K^{T}=\left[\begin{array}{c}k_{1} / l_{1} \\ 0 \\ 0 \\ 0 \\ k_{0} / l_{1}\end{array}\right]$

where $\dot{\sigma}=x_{1}-r$. It is obvious that $(A, B)$ is controllable (and stabilizable), and $A-B K$ is Hurwitz, i.e., all the eigenvalues $\lambda_{i}$ of $A-B K$ always have negative real parts, $\operatorname{Re}\left(\lambda_{i}\right)<0$, for the 
control gains $\left(k_{\mathrm{o}} / l_{1}\right.$ and $\left.k_{1} / l_{1}\right)$ and all steam generator parameter values given in Table 1 .

\subsection{A Robust Estimator for the Level Control System}

To implement the proposed optimal algebraic controller (17), the error signal $e_{1}$ must be available online. However, $e_{1}$ is not a measurable quantity since the state variable $x_{1}$ is not directly measurable. Having $e_{1}$ available online, an estimator can be designed to estimate the state variables. For this aim a robust nonlinear estimator by using the reduced-order model (3) is proposed as follows

$$
\left\{\begin{array}{l}
\dot{\hat{x}}_{1}=K_{1}(u-q)+\delta \operatorname{sat}(e) \\
\dot{\hat{x}}_{2}=-\frac{1}{\hat{\tau}_{2}} \hat{x}_{2}-\frac{\hat{K}_{2}}{\hat{\tau}_{2}}(u-q) \\
\hat{y}=\hat{x}_{1}+\hat{x}_{2}
\end{array}\right.
$$

where $\left(\hat{x}_{1}, \hat{x}_{2}, \hat{y}\right)$ are the estimations of $\left(x_{1}, x_{2}, y\right)$, respectively, the output error $e$ is $e=y-\hat{y}$, and $\delta$ is the constant estimator gain, and the parameters $\widehat{K}_{2}$ and $\hat{\tau}_{2}$ are the nominal values of the corresponding parameters in Table 1 . The parameters $\hat{\tau}_{2}$ and $\widehat{K}_{2} / \hat{\tau}_{2}$ are selected as the $5 \%$ values of $\tau_{2}(\theta)$ and $K_{2} / \tau_{2}(\theta)$ given in Table 1 in order to account for the reverse dynamic characteristics (the swell-shrink phenomenon). The saturation function is defined by

$$
\operatorname{sat}(e)=\left\{\begin{array}{ccc}
e /|e| & \text { if } & |e|>1 \\
e & \text { if } & |e| \leq 1
\end{array}\right.
$$

The robust estimator can accommodate large parameter variations and modeling uncertainties. The region of the statespace in which (20) is asymptotically stable can be determined using Lyapunov's stability theorem. For this aim, a candidate Lyapunov function can be selected as

$$
V(e)=e^{2} / 2, e=y-\hat{y}
$$

Using (3) and (20), the time derivative of $V(e)$ is found as

$$
\begin{aligned}
\dot{V}(e) & =e \dot{e} \\
& \leq|e| \cdot\left(\left|-\frac{1}{\tau_{2}} x_{2}+\frac{1}{\hat{\tau}_{2}} \hat{x}_{2}-\frac{K_{2}}{\tau_{2}}\left(u-K_{4} q\right)+\frac{\hat{K}_{2}}{\hat{\tau}_{2}}(u-q)\right|-\delta\right)
\end{aligned}
$$

If the gain $\delta$ is selected large enough, i.e. $\delta>\mid-\frac{x_{2}}{\tau_{2}}+\frac{\hat{x}_{2}}{\hat{\tau}_{2}}-$ $\frac{K_{2}\left(u-K_{4} q\right)}{\tau_{2}}+\frac{\widehat{R}_{2}(u-q)}{\hat{\tau}_{2}} \mid$, the asymptotic stability of the state estimation can be achieved since the time-derivative of $V(e)$ will be $\dot{V}<0$. Consequently, by choosing the estimator gain $\delta$ large enough, the estimation error approaches to zero as time increases. With the usage of the robust nonlinear estimator (20), the proposed optimal algebraic controller described by (17) is now suitable for practical implementation because it makes the error signal $e_{1}$ available online. The block diagram of the proposed control system is given in Fig. 2.

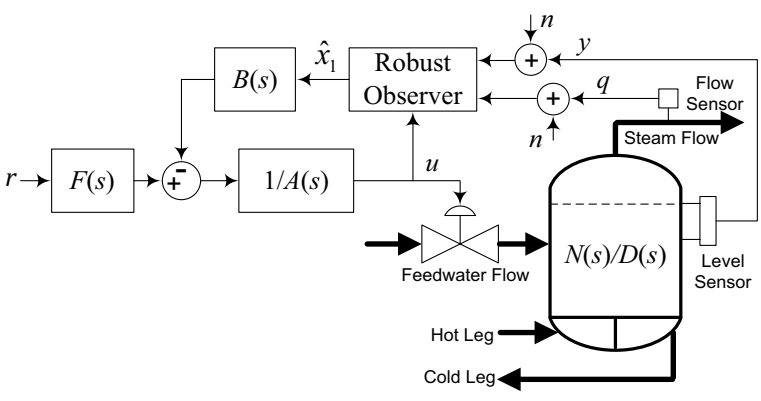

Fig. 2. Block diagram of the robust estimator-based optimal algebraic control strategy.

\section{Numerical Results}

The performance of the proposed level control system for steam generators is evaluated with a set of simulations using MATLAB for $\delta=2, \hat{\tau}_{2}=48.4$ and $\widehat{K}_{2}=9.63$ by considering parameter values at $5 \%$ power level. Numerical simulations are given for step water reference changes, and ramp power (steam) demand variations. The robustness of the proposed methodology is illustrated under power demand variations, parameter uncertainties and noisy measurements.

Figure 3 shows the response of the water level for step water reference variations. The step response of the plant is given for step reference water level changes at $5 \%, 15 \%, 30 \%, 50 \%$ and $100 \%$ power level. The effect of the negative thermodynamic behavior (swell-shrink phenomenon) can be seen at low power levels clearly, i.e. level decrease initially for power level less than or equal to $15 \%$. A non-overshoot transient response is seen in the figure as desired in the plant specifications for all power operations.

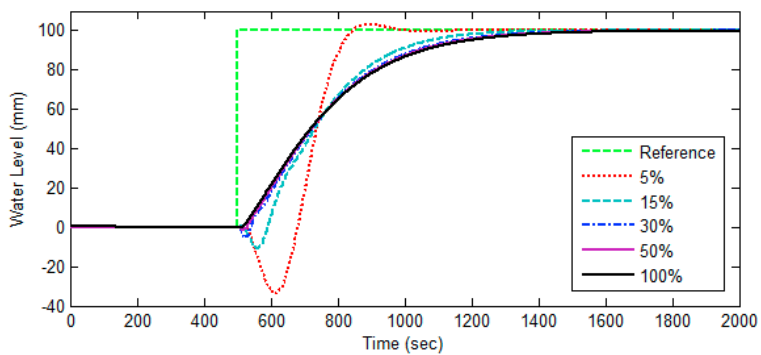

Fig. 3. Steam generator water level (in $\mathrm{mm}$ ) variations as a function of time during step reference water level variations.

The robustness and performance of the proposed control under parameter variations and noisy measurements are given in Fig. 4. When the power demand (or steam flow demand) varies in the full operating range (from $0 \%$ to $100 \%$ power level), the time-responses of the plant output (water level) and the feedwater flow input are demonstrated. During the assumed power demand (in \% rated power) variations (Fig. 4a), the feedwater flow behavior is displayed in Fig. 4b, and water level response is displayed in Fig. 4c. The noise on the water level measurement channel is assumed to be normally distributed with a mean of $0 \mathrm{~mm}$ and a standard deviation of $\pm 10 \mathrm{~mm}$. The noise on the steam flow measurement channel is assumed to be normally distributed with a mean of $0 \mathrm{~kg} / \mathrm{s}$ and a standard 
deviation of $\pm 13.4 \mathrm{~kg} / \mathrm{s}$. The narrow range reference water level is assumed to be $300 \mathrm{~mm}$. Figure $4 \mathrm{~b}$ shows that the feedwater flow rate (in $\mathrm{kg} / \mathrm{s}$ ) which is the control signal follows perfectly the steam flow rate (in $\mathrm{kg} / \mathrm{s}$ ) over all the operating power ranges during power demand variations. Figure $4 \mathrm{c}$ shows that the water level stays very close to the reference level during transients and stays on the reference signal when power demand stays constant. Even though steam generator model has model and parameter uncertainties, and noisy measurements, it is clear from figures that the robust estimator based optimal algebraic control provides excellent results. A quite robust control response is obtained against the plant uncertainties and noise.
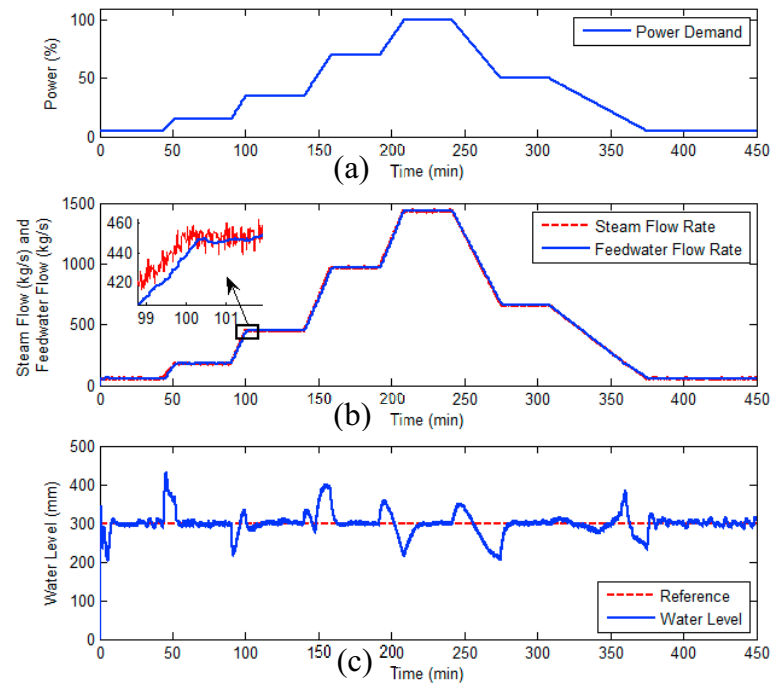

Fig. 4. Robustness and performance of the proposed level control system under parameter uncertainties and noisy measurements. (a) Power demand (in \%) variations, (b) steam flow rate (in $\mathrm{kg} / \mathrm{s}$ ) and feedwater flow rate (in $\mathrm{kg} / \mathrm{s}$ ), (c) water level (in $\mathrm{mm}$ ) during power demand variations as a function of time.

\section{Conclusion}

A robust estimator-based optimal algebraic control strategy is developed for the steam generator level control system. It is shown that the proposed control strategy is capable of efficiently controlling the steam generator level system in the presence of the noise, and modeling and parameter uncertainties with the following features:

- Efficiency: ability to handle operational maneuvers with small water deviation from reference,

- A single automatic control over the complete range of normal power operation: the conventional single-element/threeelement control system is reduced to a single control system,

- Robustness: ability to address power demands with small impact to control system stability and desired performance.

\section{References}

[1] M. Irving, C. Miossec, and J. Tassart, "Towards efficient full automatic operation of the PWR steam generator with water level adaptive control," in Proc. 2nd Int. Conf. Boiler Dynamics and Control in Nuclear Power Stations, U.K., 1979.
[2] S. K. Menon and A. G. Parlos, "Gain-scheduled nonlinear contro of U-tube steam generator water level," Nuclear Science and Engineering, vol. 111, pp. 294-308, 1992.

[3] W. Dong, J. M. Doster, and C. W. Mayo, "Steam generator control in nuclear power plants by water mass inventory," Nuclear Engineering and Design, vol. 238, p. 859, 2008.

[4] C. Liu, F. Zhao, P. Hu, S. Hou, and C. Li, "P controller with partial feed forward compensation and decoupling control for the steam generator water level," Nuclear Engineering and Design, vol. 240, no. 1, pp. 181-190, 2010.

[5] Z. Zhang and L.-S. Hu, "Performance assessment for the water level control system in steam generator of the nuclear power plant," Annals of Nuclear Energy, vol. 45, pp. 94-105, Jul. 2012.

[6] J. J. Sohn and P. H. Seong, "A steam generator model identification and robust $H \infty$ controller design with v-gap metric for a feedwater control system," Annals of Nuclear Energy, vol. 37, no. 2, pp. 180-195, Feb. 2010.

[7] M. Kim, M. Shin, and M. Chung, "A gain-scheduled L2 control to nuclear steam generator water level," Annals of Nuclear Energy, vol. 26, pp. 905-916, 1999.

[8] Y. J. Lee, S. J. Oh, W. Chun, and N. J. Kim, "The model predictive controller for the feedwater and level control of a nuclear steam generator," Nuclear Engineering and Technology, vol. 44, no. 8, pp. 911-918, Dec. 2012.

[9] Z. Dong, X. Huang, and J. Feng, "Water-level control for the Utube steam generator of nuclear power plants based on output feedback dissipation," IEEE Transactions on Nuclear Science, vol. 56, no. 3, pp. 1600-1612, 2009.

[10] A. M. H. Basher and J. March-Leuba, "Development of a robust model-based water level controller for U-tube steam generator,' Oak Ridge National Lab. Report, ORNL/TM-2001/166, 2001.

[11] M. G. Na, S. P. Kim, W. K. Chung, K. J. Lee, J. S. Song, H. C. No, and J. Y. Lee, "A PC-based water level controller for nuclear steam generators," Annals of Nuclear Energy, vol. 21, no. 10, pp. 589 600, Oct. 1994.

[12] S. R. Munasinghe, M. Kim, and J. Lee, "Adaptive neurofuzzy controller to regulate UTSG water level in nuclear power plants," IEEE Trans Nuc Sci, vol. 52, no. 1, pp. 421-429, 2005.

[13] Z. Ahmad, "Data-driven controller of nuclear steam generators by set membership approximation," Annals of Nuclear Energy, vol 37, no. 4, pp. 512-521, 2010.

[14] L. Wei, F. Fang, and Y. Shi, "Adaptive backstepping-based composite nonlinear feedback water level control for the nuclear U-tube steam generator," IEEE Transactions on Control Systems Technology, vol. Early Access Online, 2013.

[15] H. Moradi, M. Saffar-Avval, and F. Bakhtiari-Nejad, "Sliding mode control of drum water level in an industrial boiler unit with time varying parameters: A comparison with $\mathrm{H} \infty$-robust control approach," Journal of Process Control, vol. 22, no. 10, pp. 1844 1855, Dec. 2012

[16] G. Ablay, "Sliding mode approaches for robust control, state estimation, secure communication, and fault diagnosis in nuclear systems," PhD Dissertation, The Ohio State University, Columbus, OH, 2012.

[17] S. Guarro, M. Yau, and M. Motamed, "Development of tools for safety analysis of control software in advanced reactors," U.S. Nuclear Regulatory Commission, NUREG/CR-6465, 1996.

[18] W. Tan, "Water level control for a nuclear steam generator," Nuclear Eng and Design, vol. 241, no. 5, pp. 1873-1880, 2011.

[19] M. G. Na and H. C. No, "Quantitative evaluation of swelling or shrinking level contributions in steam generators using spectrum analysis," Annals of Nuc En, vol. 20, no. 10, pp. 659-666, 1993.

[20] C. T. Chen, Linear System Theory and Design. New York: Oxford University Press, 1999.

[21] S. Manabe, "Unified interpretation of classical, optimal, and Hinf control," J. of SICE, vol. 30, no. 10, pp. 941-946, 1991.

[22] Y. C. Kim, L. H. Keel, and S. P. Bhattachayya, "Transient response control via characteristic ratio assignment," IEEE Trans on Auto Control, vol. 48, no. 12, pp. 2238-2244, 2003.

[23] A. V. Lipatov and N. I. Sokolov, "Some sufficient conditions for stability and instability of continuous linear stationary systems," Automat.Remote Contr., vol. 39, pp. 1285-1291, 1979. 